\title{
L'inspection dans le système éducatif allemand
}

\author{
Ingeborg Christ
}

\section{OpenEdition}

Journals

Édition électronique

URL : http://journals.openedition.org/ries/3559

DOI : 10.4000/ries.3559

ISSN : 2261-4265

\section{Éditeur}

Centre international d'études pédagogiques

\section{Édition imprimée}

Date de publication : 1 décembre 1995

Pagination : 133-140

ISSN : 1254-4590

\section{Référence électronique}

Ingeborg Christ, «L'inspection dans le système éducatif allemand », Revue internationale d'éducation de Sèvres [En ligne], 08| 1995, mis en ligne le 14 février 2014, consulté le 01 mai 2019. URL : http:// journals.openedition.org/ries/3559 ; DOI : 10.4000/ries.3559

Ce document a été généré automatiquement le 1 mai 2019.

(c) Tous droits réservés 


\title{
L'inspection dans le système éducatif allemand
}

\author{
Ingeborg Christ
}

1 Les corps d'inspection en Allemagne ont actuellement de nombreuses fonctions. Ils interviennent au niveau de l'ensemble du système éducatif aussi bien qu'au niveau de l'établissement scolaire. Ils s'occupent de l'organisation scolaire et assurent la qualité de l'enseignement des différentes matières et des examens, en particulier de l'Abitur' ${ }^{1}$. Ils supervisent également le personnel enseignant.

2 L'inspection contrôle, inspecte et conseille. Des recherches empiriques ont démontré que la multiplicité de ces fonctions laisse peu de temps pour exercer celle à laquelle les inspecteurs eux-mêmes tiennent le plus, le conseil aux personnels des établissements. Les enseignants, de leur côté, expriment le besoin d'être conseillés bien davantage et à un autre moment que celui de l'inspection, c'est-à-dire au moment de la notation, ce qui ne se fait pas toujours sans conflits.

Depuis un certain temps, se pose donc la question du rôle et de la fonction de l'inspection et de ses conditions de travail dans un contexte où des moyens supplémentaires lui seraient nécessaires pour répondre aux besoins des enseignants. À cela, s'ajoute actuellement un deuxième débat qui risque de conduire à un changement en profondeur du rôle même de l'inspection. Il s'agit des réformes qui s'annoncent actuellement dans le système scolaire à partir des notions d'autonomie des établissements scolaires, d'efficacité du système et d'évaluation. L'inspection correspondra-t-elle encore aux exigences du nouveau système? Comment faudra-t-il concevoir ses fonctions et ses tâches pour qu'elle puisse aider à répondre aux besoins du monde actuel?

\section{Situation actuelle}

4 L'inspection relève de la compétence des Länder. Précisons qu'il y a seize ministres de l'éducation en Allemagne. Pour empêcher que ne se créent des divergences trop grandes, les Länder se sont dotés d'un instrument de coordination, la conférence permanente des 
ministres de l'éducation, qui se réunit en séance plénière quatre fois par an. Ses décisions, préparées par des commissions de travail permanentes, sont votées à l'unanimité et lient les Länder. Chacun des ministres garantit la qualité de l'enseignement dans son Land, mais dans le cadre des décisions communes qui sont observées scrupuleusement. Ce principe est particulièrement important en ce qui concerne l'Abitur, qui autrement risquerait de ne pas être reconnu dans les différents Länder.

Dans chacun des seize Länder la dénomination des ministres de l'éducation varie selon les traditions et leurs domaines de compétence. Le ministre fixe les principes de l'éducation dans le Land, garantit la programmation et le fonctionnement du système et décide en cas de litige. Dans la plupart des Länder, une partie de ses compétences est déléguée à des instances intermédiaires et à des instances locales auxquelles sont rattachés les inspecteurs. L'instance intermédiaire est l'inspection supérieure pour les écoles. Les inspecteurs ont un contact direct avec les établissements scolaires respectifs. Pour l'école élémentaire et une partie des écoles du premier cycle secondaire, l'inspection est déléguée à l'inspection inférieure qui relève du niveau de la commune.

Comme la Rhénanie-Nord-Westphalie est un Land géographiquement important (10\% à peu près du territoire et $20 \%$ de la population de la République) et avec un nombre élevé d'écoles, on y trouve tous les niveaux de l'inspection. D'autres Länder, où le nombre d'établissements scolaires est plus réduit, - par exemple les villes-États de Brême et d'Hambourg - réunissent les deux niveaux supérieurs en un seul. La Bavière, un Land très étendu également, délègue traditionnellement une partie de l'inspection, notamment le contrôle du personnel, aux directeurs des établissements auxquels sont conférés des tâches spéciales. Une partie de la programmation incombe en Bavière à un institut pédagogique du Land (Staatinstitut für Schulpädagogik - ISP).

\section{Les fonctions de l'inspection}

7 Les établissements disposent à l'heure actuelle d'une autonomie relativement grande dans le domaine de l'organisation, du fonctionnement et du déroulement de la vie scolaire, en s'appuyant toutefois sur les décrets et prescriptions des organismes supérieurs. Leur autonomie ne concerne actuellement ni le budget ni l'embauche des enseignants. L'inspection se veut en général discrète, considérant que sa fonction est plutôt de conseiller et de coopérer que de diriger. Ceci n'exclut pas que les établissements et les enseignants, eux, se plaignent parfois d'une trop forte pression due aux décrets et aux règlements.

8 À chaque niveau, l'inspection est constituée d'un collectif de pédagogues, d'administrateurs et de juristes. Dans sa totalité, elle représente l'obligation et le droit de l'État de garantir l'organisation, la programmation, la direction et le contrôle du système scolaire. Dans une société pluraliste et démocratique, ce devoir et cette obligation exigent qu'un consensus soit sans cesse établi, à l'intérieur de l'inspection elle-même ainsi qu'entre l'inspection et les établissements.

Les fonctions de l'État s'exercent dans trois domaines.

10 Au plan juridique, il contrôle et garantit que les droits de tous les partenaires de l'établissement et du système scolaire - ceux des élèves et de leurs parents et ceux des enseignants - sont respectés. Dans le domaine du service, il contrôle et garantit 
l'accomplissement des devoirs de la part du personnel enseignant. Enfin, il contrôle et garantit la qualité de l'enseignement dispensé.

11 Les deux premiers domaines concernent, en grande partie, des aspects juridiques et administratifs. Le troisième domaine est davantage caractérisé par des aspects pédagogiques et disciplinaires.

12 L'élaboration de nouveaux programmes d'enseignement des disciplines constitue un exemple des liens étroits qui existent entre les différents domaines. En Allemagne, on part du principe que les programmes sont valables pour une durée approximative de dix ans. En fonction de cette durée, leur élaboration et leur mise en œuvre se font donc avec le plus grand soin. Notons au passage qu'en règle générale, il faudrait plutôt parler de directives que de programmes, étant donné que leur caractère est moins concret et moins obligatoire que les programmes français, et cependant plus concret et plus obligatoire qu'un simple cadre.

13 Le moment venu, quand des changements dans la société, le développement dans les sciences ou dans les conceptions de l'éducation l'exigent, le ministère de l'éducation envisage d'élaborer de nouveaux programmes. Il propose une structure globale qui sert de base de travail pour toutes les matières et instaure pour chacune une commission constituée par des enseignants expérimentés, des représentants des instituts pédagogiques, un représentant du secteur des études supérieures et, comme directeur et coordonnateur du travail de chaque commission, un inspecteur de la discipline concernée.

14 Le ministre de l'éducation suit et coordonne les travaux, s'informe des problèmes des différentes commissions, veille au respect de la planification temporelle et publie les textes. Il veille à ce que les parties concernant les décisions de la conférence permanente de l'éducation, surtout celles qui se réfèrent à l'Abitur, soient respectées et insère les passages respectifs au texte. Les manuscrits, avant publication, sont présentés, dans le cadre de la participation, aux associations de parents, aux syndicats d'enseignants, au Parlement du Land, etc. pour garantir le respect des lois et des droits. Après leur publication, les inspecteurs sont chargés d'expliquer les principes pédagogiques du programme dans des réunions de directeurs d'établissement, et les principes méthodologiques dans des réunions avec les enseignants des différentes disciplines.

\section{Le travail des inspecteurs}

Les tâches de chaque inspecteur se situent dans trois domaines: l'établissement, sa discipline, d'autres domaines spécifiques.

En Rhénanie-Nord-Westphalie, chaque inspecteur est responsable d'un nombre déterminé d'établissements. Il doit s'intéresser à la situation du personnel, connaître les difficultés pédagogiques et y remédier en cas de difficultés et assurer la mutation des professeurs, s'il en est besoin. Il est un partenaire du directeur de l'établissement, mais il doit en même temps veiller au respect du règlement. En cas de nécessité, il est la personne que peuvent contacter les enseignants, les parents et les élèves. Il doit aussi parfois protéger les enseignants contre des exigences injustifiées des parents. Il inspecte et note les enseignants et fait des propositions pour leur promotion. Il est censé connaître le travail en classe et a le droit d'assister aux examens oraux de l'Abitur. Il doit 
s'intéresser à la vie scolaire et encourager projets et expériences. Par sa présence, par ses conseils et sa participation, il peut contribuer à une vie pédagogique active.

L'inspecteur est responsable de sa discipline pour toute la région. Il contrôle les sujets des examens écrits de l'Abitur pour sa matière. Il assure l'égalité du niveau en comparant les copies des examens des différents établissements ${ }^{2}$. L'inspecteur coopère avec les instituts de formation continue, en suggérant des thèmes ou en contribuant lui-même à la formation, et il invite les professeurs de sa discipline pour des échanges d'expériences. Il coordonne le travail de la commission des programmes ainsi que les expériences scolaires menées dans sa discipline. Dans ce travail, il est assisté de plusieurs enseignants déchargés d'une partie de leurs obligations scolaires (conseillers de la discipline Fachberater).

Enfin, chaque inspecteur a des tâches spécifiques situées également au niveau de la région. Ainsi, il y a un spécialiste des questions concernant le premier cycle de l'enseignement secondaire; un autre s'occupe spécialement du second cycle; un troisième doit être contacté en cas de problèmes lors des examens de l'Abitur. Tel autre s'occupe des questions de l'intégration des élèves étrangers, tel autre de celle des élèves handicapés. L'un connaît tous les règlements concernant les voyages d'études des élèves et les rencontres internationales, tandis qu'un autre s'occupe d'informer les écoles sur des concours extrascolaires concernant les élèves...

Cette description du travail des inspecteurs peut paraître idéale, mais elle n'est pas pour autant erronée. S'il est vrai que toutes les tâches ne peuvent pas être réalisées avec la même perfection, vu leur grand nombre, il n'en reste pas moins qu'elles devraient l'être parce que toutes ont une grande importance pour le bon fonctionnement du système scolaire.

\section{Le recrutement des inspecteurs}

Le recrutement se fait par la mise au concours d'un poste dans le bulletin officiel. Pour pouvoir poser sa candidature, il faut avoir passé les deux examens d'État prescrits pour être enseignant en Allemagne. Il faut en plus une grande expérience comme enseignant et, en règle générale, comme directeur d'une école ou, dans des cas moins fréquents, comme conseiller pédagogique, c'est-à-dire comme formateur de professeurs-stagiaires. La rémunération est identique à celle du directeur des lycées (Gymnasium).

21 Il n'y a pas de formation spéciale des inspecteurs. Ceux-ci ont pris eux-mêmes des initiatives en instituant des associations qui invitent leurs membres à des réunions de formation. Le ministère, de son côté, réunit tous les ans les inspecteurs pour coordonner le travail et débattre des sujets posant problème.

\section{Perspectives d'avenir}

22 Les projets de réforme actuels suscitent un nouveau débat qui ne porte plus sur le système éducatif en général mais sur une nouvelle vision du rôle de l'établissement scolaire. On observe à l'heure actuelle des changements dans la structure de la société qui ne manqueront pas de toucher le système scolaire et, par conséquent, le rôle et les fonctions de l'inspection. 
23 Le point de départ des réflexions sur cette nouvelle réforme sont les défis du monde moderne, auxquels l'école doit répondre mais auxquels elle ne réagit pas toujours aussi efficacement qu'il serait souhaitable, les structures étant parfois trop rigides. Les reproches adressés au système scolaire sont d'abord d'ordre pédagogique. Il est rendu responsable d'un manque de motivation des élèves aussi bien que des enseignants. Un autre reproche est plutôt d'ordre économique; on se demande si, dans le domaine de l'enseignement, la relation coûts-effets est toujours optimale.

Une part de responsabilité est attribuée à l'inspection à laquelle on reproche de réagir trop lentement aux problèmes des écoles et des enseignants, et par des mesures qui ne correspondent pas toujours aux exigences locales.

Ces remarques ont conduit à s'intéresser à des expériences menées dans le secteur de l'économie, expériences selon lesquelles une plus grande décentralisation ne signifierait pas seulement plus de démocratie mais mènerait aussi à une plus grande motivation des partenaires et, en fin de compte, à une plus grande efficacité. Dérégulation et distribution des responsabilités sont devenues aussi, depuis un certain temps, les lignes directrices d'une administration publique moderne et deviennent également, de plus en plus, des mots-clés pour l'avenir scolaire. On peut sans doute prédire qu'une école plus autonome pourra réagir d'une façon plus souple à des problèmes locaux. Elle susciterait également plus de créativité parmi le personnel enseignant et chez tous ceux qui participent à la vie de l'établissement. C'est pourquoi on s'attend à ce que la vie et le travail scolaires donnent davantage de satisfaction. Enfin, selon les expériences menées dans le secteur économique, on peut espérer qu'il en résultera une plus grande efficacité ainsi qu'une meilleure utilisation des ressources personnelles et matérielles. Pour les enfants, une telle école serait un lieu où ils pourraient non seulement apprendre mais vivre l'expérience d'une vie démocratique de la coopération, du jeu de toutes les forces qui participent à la vie scolaire, de la recherche d'un consensus et de l'exercice de responsabilités. Le résultat qu'on espère atteindre est l'identification avec le système local où ils vivent.

Si tel est l'avenir du système scolaire, pour l'inspection la question est de savoir comment elle pourra contribuer à l'évolution de l'école. Quelle structure l'inspection devra-t-elle adopter et comment devra-t-elle organiser son travail pour être à même de l'aider à répondre aux exigences et aux possibilités du monde moderne actuel ?

Les raisons d'une efficacité insuffisante ont été identifiées: une centralisation trop grande, des décisions tombant trop loin du lieu de l'action, trop peu de stimulants pour une activité responsable.

28 Pour garantir une amélioration, les remèdes suivants semblent possibles: une mobilisation plus grande des forces propres, une motivation pour l'engagement, plus de responsabilité pour chaque école, plus de coopération entre les partenaires (enseignants, élèves, parents).

29 L'école, pour résumer le défi, devra devenir la " propriété » de ceux qui y sont engagés, propriété qu'il faut protéger, cultiver et améliorer. On peut certes se demander, si dans les années 1970, on n'a pas déjà, pour garantir la participation des partenaires, publié maints décrets qui ont entraîné de nombreux résultats positifs. La différence est que, à l'heure actuelle, la participation, dans une école plus autonome, est censée devenir plus intense, plus sérieuse aussi et plus conséquente. 


\section{Les limites de l'autonomie}

30 Avec le développement d'une plus grande autonomie se pose en même temps la question de la responsabilité de l'État pour l'enseignement. La Constitution de la République fédérale d'Allemagne n'autorise pas qu'on laisse la responsabilité totale de l'enseignement aux écoles elles-mêmes. Le système scolaire est soumis à l'autorité de l'État, c'est ce qui est formulé clairement dans la Loi fondamentale dont l'article 72 exige la garantie de l'égalité de la vie pour tous les citoyens. C'est une tâche dont l'État ne peut se défaire. Il est vrai qu'une autonomie plus grande peut être accordée aux écoles mais il faut que les normes et les standards requis soient garantis ainsi que l'équivalence des examens, par exemple, celle de l'Abitur. En plus, il est nécessaire que soient garanties l'égalité des ressources pour les écoles et les valeurs prescrites par la constitution.

31 Dans tous ces domaines, l'État, représenté par l'inspection, est appelé au contrôle. Il s'ensuit que, si la situation des établissements change, la relation école/inspection devra être redéfinie. L'inspection devra trouver l'équilibre entre les directives centrales qui garantissent l'homogénéité du système scolaire, d'une part et la décentralisation des compétences en faveur des écoles, d'autre part. Elle devra trouver le moyen de garantir l'équivalence dans la pluralité et l'individualité.

32 Si l'inspection ne veut ou ne doit plus donner de directives elle-même, elle sera néanmoins appelée à aider les écoles à utiliser leurs libertés et leurs possibilités de conférer une forme individuelle à la vie de l'établissement, sans pour autant mettre en péril les normes et la qualité requises pour l'enseignement.

33 Les moyens qui sont proposés jusqu'ici tournent autour de notions comme " programmes scolaires » et "évaluation». Un programme scolaire est une tâche pédagogique et éducative à laquelle s'engagent les partenaires de l'enseignement en tenant compte des directives prescrites d'une part, et en mettant à profit les marges de libertés pédagogiques garanties par les mêmes directives, d'autre part. Un programme né de la sorte d'un accord commun est l'expression de la philosophie d'un établissement et de ses exigences ainsi que de ses possibilités. Il représente, dans le meilleur des cas, un consensus et le vœu d'une responsabilité commune pour le bien des jeunes.

34 Un programme scolaire conçu ainsi a besoin d'une évaluation interne pour s'assurer que les objectifs ont été atteints. Il a également besoin d'une évaluation externe. L'inspection, selon les propositions actuelles, aura une mission plus importante dans l'élaboration des techniques d'évaluation interne et externe. Tout d'abord, elle aidera les établissements à créer leur programme et à l'évaluer eux-mêmes. Ensuite, elle donnera le feed-back et aidera à discerner les points faibles ou les points forts, selon les cas, offrant, si besoin est, le support du conseil ou parfois, aussi, les moyens d'une formation continue.

Quant à l'évaluation interne, les participants devront être mis à même de constater quels objectifs ont été atteints et devront savoir se poser des questions sur leur travail et y réfléchir. L'inspection contribuera à ce processus de réflexion, le directeur de l'établissement également.

36

L'inspection aura donc moins la tâche de juger ponctuellement le travail de l'enseignant, de le valoriser ou de le noter. Le travail en classe jouera, bien sûr, toujours un rôle prépondérant, mais il sera considéré davantage comme une partie d'un tout beaucoup plus vaste et plus complexe qu'on ne pourra pas séparer de l'ensemble, ni juger 
ponctuellement. De toute façon, on compte accorder à l'avenir, en Allemagne, un rôle plus important qu'actuellement au chef d'établissement pour noter les enseignants. On pense en effet qu'il est le mieux à même d'évaluer la contribution de l'individu au travail commun.

Les tâches de la nouvelle inspection seront caractérisées par les mots-clés suivants: conseil plutôt que contrôle ; contribution au développement de l'établissement au profit du développement du système scolaire; aide à la capacité de résoudre des problèmes d'enseignement dans un établissement jouissant de plus d'autonomie ; contribution au développement du sens des responsabilités chez les partenaires; encouragement à plus d'autonomie de chaque établissement ; élaboration de concepts d'une évaluation interne et externe de la qualité et de l'efficacité du système.

Une nouvelle conception de l'inspection n'éliminera pas tous les problèmes. L'indépendance et l'autonomie peuvent entraîner des échecs.

Dans une situation financière difficile, comme c'est le cas actuellement, autonomie peut signifier pénurie, situation désagréable et peu motivante qui risque d'engendrer la méfiance envers des projets de réforme. Parfois, les enseignants craignent d'une part, la prise de décision en équipe, le travail commun et les discussions avec d'autres partenaires, d'autre part, un engagement plus prenant dans la vie de l'établissement et un surcroît de travail. On peut redouter également que des travaux relevant de la compétence des collectivités territoriales ou de l'administration ne reviennent à l'établissement dès lors qu'il est plus autonome. L'implication des parents dans la vie scolaire de leurs enfants devra être plus forte et les chefs d'établissement redoutent l'intrusion d'opinions non professionnelles dans le travail pédagogique. Ils regretteront le temps où ils pouvaient s'appuyer sur l'inspection qui, de son côté, est parfois préoccupée par la question de savoir si les standards pourront vraiment être garantis. Quelques sceptiques finalement se demandent si ce n'est pas un contresens en soi de vouloir imposer l'autonomie.

Quoi qu'il en soit, la place et la fonction de l'inspection dans le système scolaire ont fait l'objet de nombreux débats. Si ces discussions réussissent à sensibiliser les partenaires de l'enseignement pour qu'ils prennent conscience de leurs responsabilités, un pas est déjà fait. L'objectif essentiel n'est pas de créer une école autonome pour elle-même, mais de contribuer au développement d'une école de bonne qualité qui réponde aux besoins du monde actuel pour le bien des enfants et des jeunes. Les expériences des autres pays nous sont utiles et c'est dans ce sens que les réflexions ci-dessus pourront peut-être servir.

\section{NOTES}

1. Équivalent du baccalauréat français (NDLR).

2. Les méthodes dans les différents Länder varient sur ce point; en règle générale, les copies sont soumises à une double ou même triple correction, l'inspection se contentant d'un contrôle au hasard et dans des cas posant problème. 


\section{RÉSUMÉS}

Chaque Land allemand dispose de corps d'inspection correspondant aux différents niveaux de la scolarité. Leurs tâches sont à la fois administratives et pédagogiques. L'évolution des établissements vers une plus grande autonomie, la nécessité de mieux évaluer l'efficacité du dispositif conduisent à redéfinir les fonctions actuelles de l'inspection et les relations de l'inspecteur avec les différents partenaires.

INDEX

Index géographique : Allemagne

Mots-clés : autonomie, établissement d'enseignement, inspection, système éducatif

\section{AUTEUR}

\section{INGEBORG CHRIST}

Inspecteur auprès du ministère du Land de Rhénanie du Nord-Westphalie, Düsseldorf, Allemagne. 